\title{
Customized Treatment Option for Malpositioned Dental Implant Placed in Aesthetic Zone
}

\author{
Priyanka N. Khungar ${ }^{1}$, Trupti M. Dahane ${ }^{2}$, Ramnath P. Revankar ${ }^{3}$, Rupali Patel ${ }^{4}$
}

\begin{abstract}
${ }^{1}$ Department of Prosthodontics, Sharad Pawar Dental College, Datta Meghe Institute of Medical Sciences, DMIMS, Sawangi (Meghe), Wardha, Maharashtra, India. ${ }^{2}$ Department of Prosthodontics, Sharad Pawar Dental College, Datta

Meghe Institute of Medical Sciences, DMIMS, Sawangi (Meghe), Wardha, Maharashtra, India. ${ }^{3}$ Department of Prosthodontics, Sharad Pawar Dental College, Datta Meghe Institute of Medical Sciences, DMIMS, Sawangi (Meghe), Wardha, Maharashtra, India. ${ }^{4}$ Department of Prosthodontics, Sharad Pawar Dental College, Datta Meghe Institute of Medical Sciences, DMIMS, Sawangi (Meghe), Wardha, Maharashtra, India.
\end{abstract}

\section{INTRODUCTION}

Replacement of single missing tooth in the aesthetic region presents a challenge for a dentist. ${ }^{1}$ Various treatment considerations have to be dealt before decision making. Treatment options for such cases are removable partial denture, conventional or resin - bonded fixed partial denture (FPD), and implant-supported fixed prosthesis. Each modality is a possible treatment option and has its advantages and disadvantages.2,3 The treatment option selected would depend on patients' age, desires, compliance, also the treatment cost, adjacent teeth conditions, bone availability and aesthetics being of prime concern. However, it is found that implantsupported crown is the most preferred option in case of single missing tooth in the aesthetic zone.2,4,5 Implants besides being advantageous over resin-bonded or traditional fixed partial dentures, they also prevent the unnecessary restoration on the adjacent sound teeth as required in an FPD. Hence, implants help in saving the integrity of the existing tooth in situations where the adjacent tooth is vital and have no restorations. ${ }^{2}$ Also, many authors have suggested implant as a predictable treatment option in case of single tooth replacement. 6,7

The success of implant is not only defined by the Osseo integration achieved but also its proper placement and with harmonious and naturally blending prosthesis. ${ }^{8}$ Yet sometimes, there may be situations wherein misaligned placement of implant and reduced interarch space for future prosthesis may pose a challenge for the Prosthodontist, especially in aesthetic zone. Methods like angulated abutments, castable abutments or in severe cases even removable prosthesis have been suggested to surmount such complications. ${ }^{4}$ The following article presents with two such case scenarios dental implants were restored with castable abutment.

Restoration of missing teeth in aesthetic zone is of concern for any individual. Treatment options like removable partial denture, fixed partial denture or implant retained prosthesis can be opted. The treatment option selected would depend on patients' age, desires, compliance, also the treatment cost, adjacent teeth conditions, bone availability and aesthetics being of prime concern.

Keeping in mind the patients' age bone and periodontal conditions, implantsupported crown is the most preferred option in case of single missing tooth in the aesthetic zone. The success of implant is not only defined by osseointegration achieved, but also its proper placement and with harmonious and naturally blending prosthesis. Yet sometimes, there may be situations wherein misaligned placement of implant and reduced interarch space for future prosthesis may pose a challenge for the prosthodontist, especially in the aesthetic zone. In such complex cases, restoration with a customized treatment plan has to be carried out in order to fulfil patients' aesthetic requirement as well as restore the implant with a fixed prosthesis. The following article presents with two such case scenarios where the misaligned dental implant placed and the other with reduced interarch space were restored with castable abutment.
Corresponding Author:

Dr. Priyanka N. Khungar.

\# 99A, Ashray,

Queta Colony,

Lakadganj, Nagpur - 440008,

Maharashtra, India.

E-mail: khungarpriyanka1@gmail.com

DOI: $10.14260 /$ jemds/2020/642

How to Cite This Article:

Khungar PN, Dahane TM, Revankar RP, et al. Customized treatment option for malpositioned dental implant placed in aesthetic zone. J Evolution Med Dent Sci 2020;9(39):2930-2934,

10.14260/jemds/2020/642

Submission 30-04-2020,

Peer Review 18-07-2020,

Acceptance 25-07-2020,

Published 28-09-2020.

Copyright (C) 2020 JEMDS. This is an open access article distributed under Creative Commons Attribution License [Attribution 4.0 International (CC BY 4.0)] 


\section{PRESENTATION OF CASE}

\section{Case 1}

A 40-year-old female patient reported to the Department of Prosthodontics, for prosthesis with implant placed in upper front region of jaw. The Intra Oral Periapical radiograph (Figure 1a) revealed an implant properly osseointegrated and gingival former placed in 14 region. The Osstem mini implant of dimension $4.5 \times 11 \mathrm{~mm}$ was placed 4 months back. Gingival former was then removed, and proper gingival collar was seen circumferentially around the implant head (Figure 1b). So, a transfer abutment of size $4.5 \times 3 \times 5.5 \mathrm{~mm}$ [Mini] was placed. But, it was found that there was limited interarch space for prosthetic components (Figure 2) and if tried to trim the abutment height it would affect the long term prosthesis retention over the implant. Hence, it dictated the use of customized abutment with screw-retained prosthetic crown for this case.

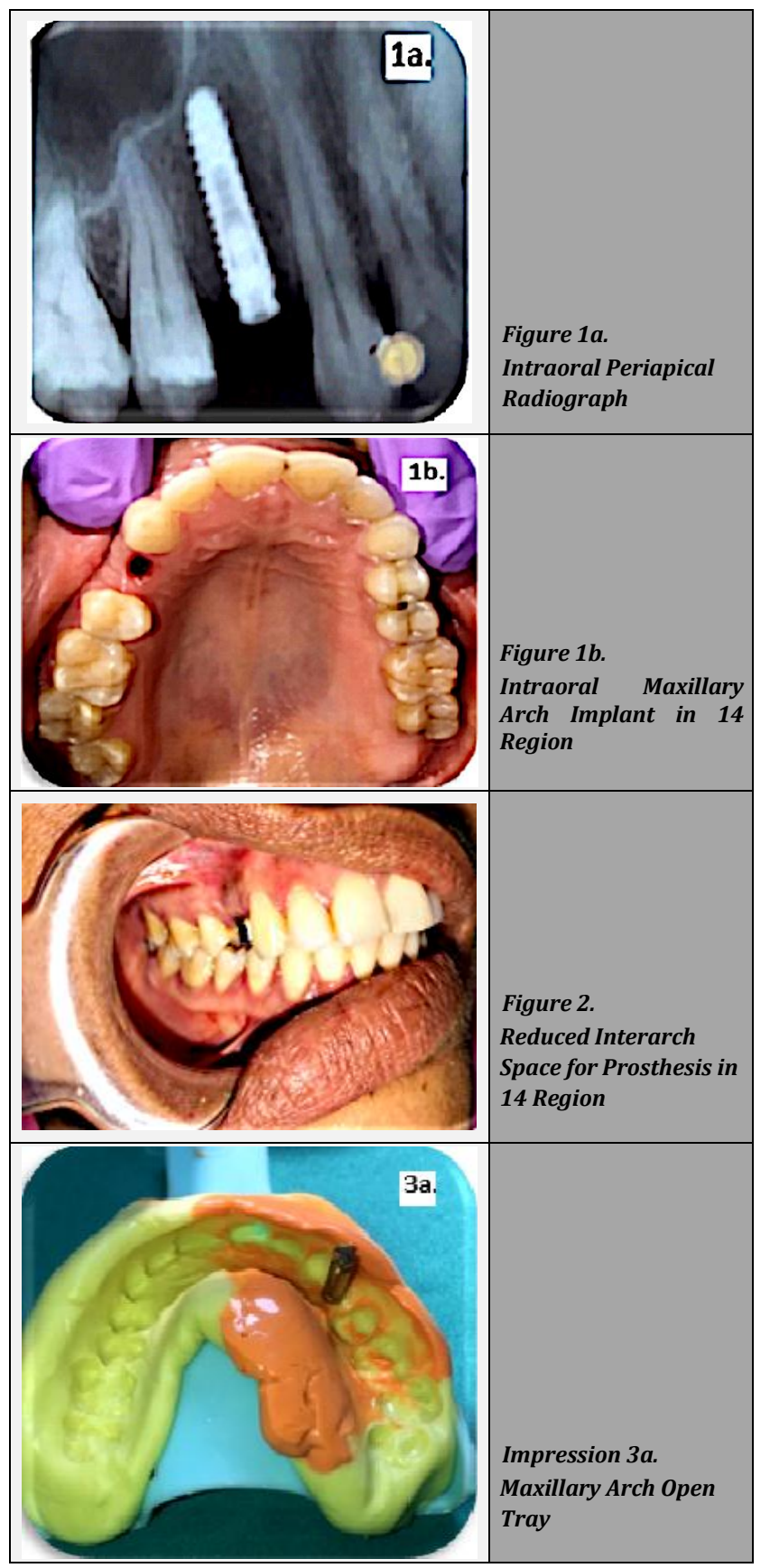

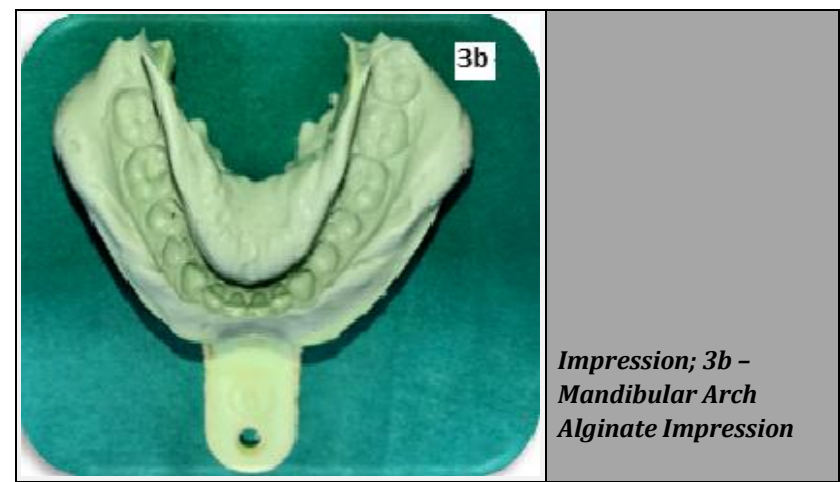

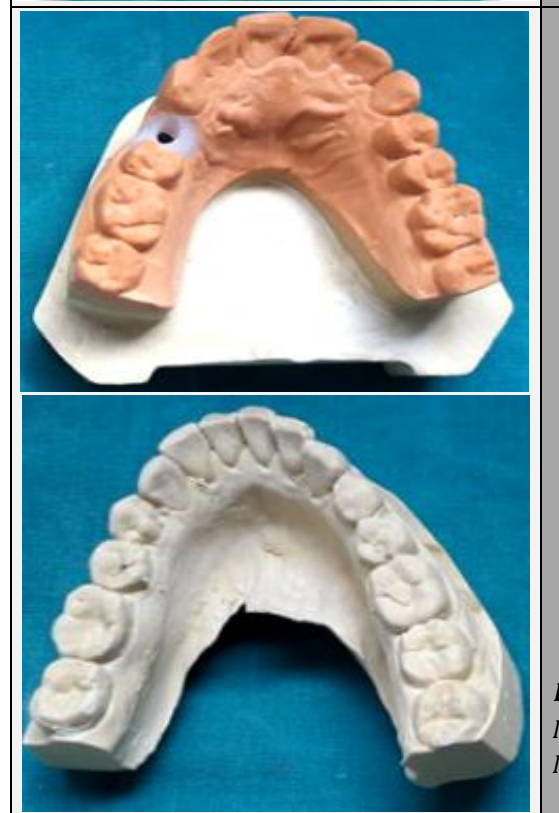

Figure 4.

Maxillary and

Mandibular Casts
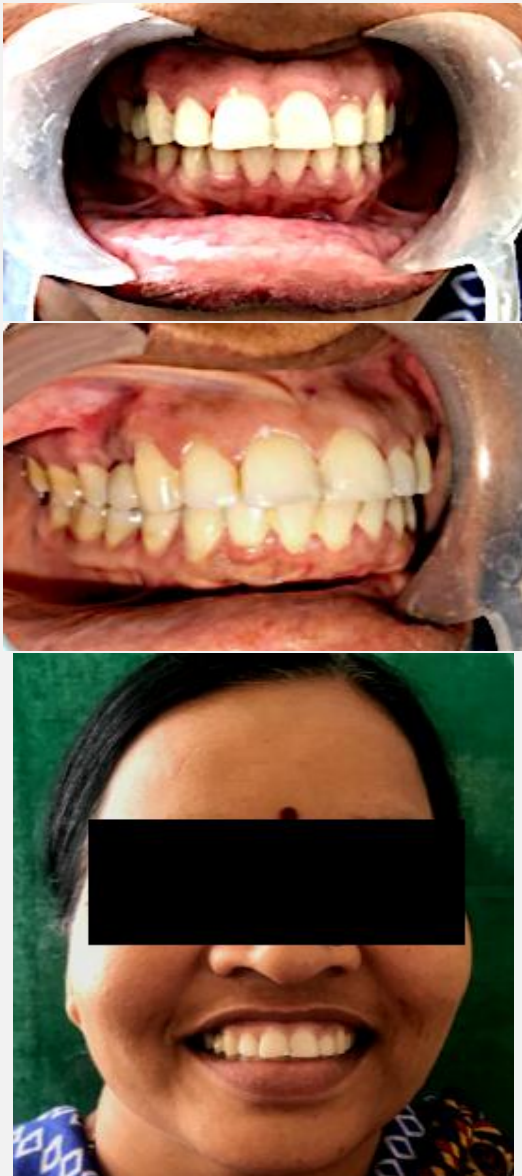

Figure 5.

Screw-Retained

Prosthesis with

Tooth 14 


\section{For Fabrication of Screw-Retained Prosthesis, Following} Steps were Carried Out

- $\quad$ Stock tray of appropriate size was selected for making implant-level open tray impression of maxillary arch and a window was made in 14 region such that Mini Fixture Pick-up Impression Coping (Osstem) can be seen to emerge out through the window without obstruction.

- Impression was then made using Addition Polysilicone (Reprosil) impression material with one stage putty wash technique, taking care that the pickup impression coping protruded out through the window that was made in tray.

- After setting, screw of impression coping was loosened, the impression was carefully removed from patients' mouth and checked for required details. Mini Fixture Lab Analog (Osstem) was then attached to the coping and screw was tightened (Figure 3a)

- Impression was poured in Type IV dental stone (Kalrock) and cast was made using soft tissue replicating material in the implant area.

- The maxillary (open tray impression) and mandibular (alginate impression - Figure 3b) casts were obtained (Figure 4).

- $\quad$ Mini NP - Cast abutment - plastic (Osstem), was then attached on the Implant lab analog and wax pattern was made according to available interarch space. The pattern was cast and tooth coloured porcelain was applied to reproduce the teeth as per the shade selected and proper contour.

- Retaining screw was placed through casted crown and threaded into the abutment. The screw - retained implant prosthesis was placed intraorally in 14 region and checked for proper seating, occlusion and aesthetics. Abutment screw was then tightened using hex up to 30 $\mathrm{Ncm}$ torque. Cotton pellet was placed over screw head and access hole on the occlusal surface was then sealed with composite resin of appropriate shade.

- At the end, aesthetic appearance and functional adaptability of the prosthesis to patient's occlusal scheme was checked (Figure 5).

\section{Case 2}

Another female patient of 46 years of age reported to department of Prosthodontics, with a mobile implant crown in upper front region of jaw. After detailed history, it was found that patient had implant-supported crown placed in 13 region of $3.3 \mathrm{~mm}$ diameter (equinox dental implant) 1 year back. Patient was unsatisfied with the fit and aesthetics of this prosthesis. She provided with older orthopantomogram that revealed deeply placed implant with same (Figure 6).

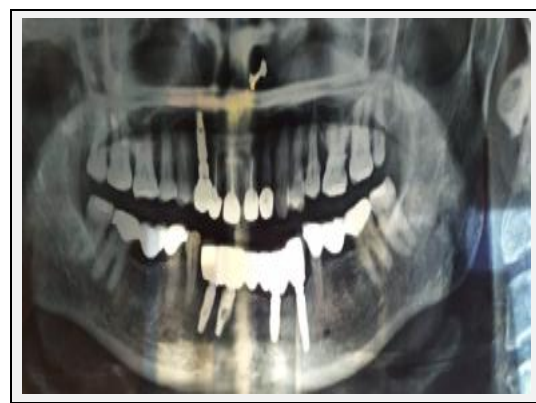

Figure-6.

Orthopantomogram Showing Deeply Placed Implant with Tooth 13

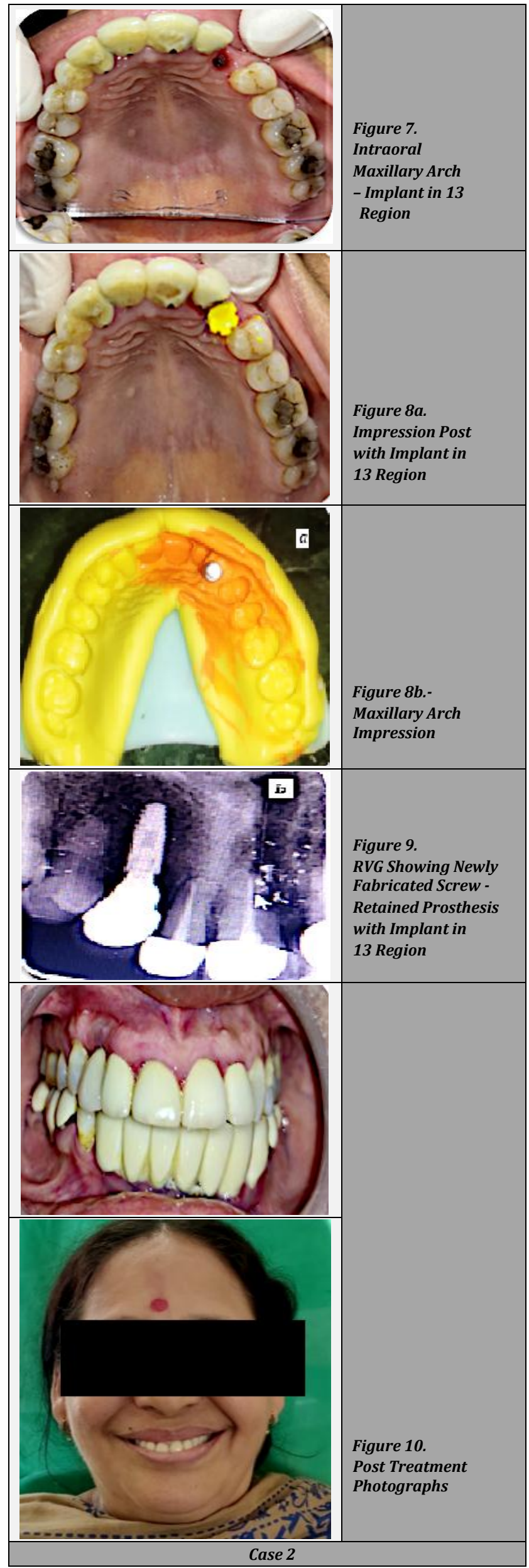


On intraoral examination, the cement retained implant prosthesis along with the abutment was removed. Gingival tissue was allowed to heal for two weeks by placing healing abutment. After two weeks a well healed gingival collar was seen around the implant (Figure 7). As the implant was misaligned with mesial tilt as well as deeply placed, screwretained prosthesis with customized abutment was planned for patient.

For impression making, closed tray impression post was placed on implant (Figure 8a) and impression was made using single step putty wash impression technique with Addition Polysilicone (Reprosil). The impression was carefully removed after setting. Then, impression post was removed from patients' mouth and implant lab analog was attached. The assembly was properly oriented and replaced in impression (Figure 8.b). The pouring of impression, fabrication of prosthesis, verifying the fit, aesthetics and occlusion of screwretained prosthesis was carried out in same manner as described in the first case (Figure 9).

Follow up was done after 1 week, 1 month and 6 months to check fit, aesthetics and patients' satisfaction with prosthesis. It was found that there was no mobility, aesthetics was optimum and patient was also satisfied with the prosthesis (Figure 10).

\section{DISCUSSION}

In both the above cases, screw-retained prosthesis with castable abutment was fabricated having customized abutments providing various advantages like retrievability, reduced chances of peri-implantitis due to residual cement, restoring optimum aesthetics in such situations where cement retained implant prosthesis is not possible.9,10 The other option like removable prostheses was not practical for single missing tooth in addition to be objectionable by the patient. Also, angulated abutments would not be of help in above cases. $^{4}$

For screw-retained prosthesis to be successful, screw should be torqued to $50-75 \%$ of their yield strength. ${ }^{10,11}$ This would ensure accurate fit between the head of the implant and abutment. Any vertical occlusal force on prosthesis will not stress the screw or cause screw loosening. However, when inaccurate castings are screwed into implants, gaps are created and vertical loading over the implant head can compress the casting and cause screw loosening ${ }^{10}$. So, care should be exercised for same.

However, there are some concerns regarding the use of screw-retained implant prosthesis which may affect the decision of using them as a treatment modality. The cost of fabricating this prosthesis is more than the cement retained one owing to the extra components needed, such as plastic sleeves, laboratory fixation screws, and the fixation screws. Nevertheless, this should be weighed against the potential costs of damaging the cemented restoration if biologic or technical complication occurs. ${ }^{11}$

Access can be difficult in the posterior regions in case of screw - retained prosthesis. So care should be taken so as to prevent screw loosening and risk of accidental aspiration of the screw by retightening of screw periodically. ${ }^{11}$

Developing harmonious occlusion can be problematic as the sealing of the screw access channel with cement may interfere with excusive or protrusive movements. Also, if not properly done it might as well lead to off-axial loading on the implants and can cause deleterious effects. Thus, a follow up including thorough examination of screw-retained prosthesis is mandatory. 11

The screw loosening is considered to be one of the major problems in screw - retained restorations. However, the frequency screw loosening reduced with the improvements in implant systems, including the advent of internal implantabutment connections, enhancement of torque drivers, and screw materials and design. But on the other hand, this screw loosening can be regarded as one of the means of assessing the prosthesis that can prevent more serious complication from occurring. ${ }^{11}$

A trend toward laboratory - customized anatomical or aesthetic abutments has emerged which offers with primary advantage of being patient specific. Customized abutment provides with another major benefit of fabricating the abutment with crown and bridge precious metal and layering the facial portion by porcelain. ${ }^{9}$

\section{CONCLUSIONS}

Aesthetic replacement of teeth ideally resembling the original tooth in contour and shade while restoring and preserving the bone and soft tissues has become an important standard for implant dentistry. This article describes the use of customized abutment with screw-retained implant prosthesis in aesthetic zone thus satisfying patient's aesthetic demand and rendering a successful treatment.

Financial or Other Competing Interests: None.

\section{REFERENCES}

[1] Kim H, Song MJ, Shin SJ, et al. aesthetic rehabilitation of single anterior edentulous space using fiber-reinforced composite. Restor Dent Endod 2014;39(3):220-5.

[2] Hebel K, Gajjar R, Hofstede T. Single-tooth replacement: bridge vs. implant- supported restoration. J Can Dent Assoc 2000;66(8):435-8.

[3] Al-Quran FA, Al-Ghalayini RF, Al-Zubi BN. Single-tooth replacement: factors affecting different prosthetic treatment modalities. BMC Oral Health 2011;11:34.

[4] Al-Fahd A, Radi I. Management of complex deeply positioned, severely mal-aligned dental implant: a case report. J Implant Adv Clin Dent 2015;7(9):31-5.

[5] Mishra SK, Chowdhary R, Patil PS, et al. Replacement of missing central incisor with single implant-supported fixed prosthesis. J Curr Res Sci Med 2016;2(2):116-9.

[6] Tallarico M, Caneva M, Baldini N, et al. Patient-centered rehabilitation of single, partial and complete edentulism with cemented- or screw- retained fixed dental prosthesis: the First Osstem Advanced Dental Implant Research and Education Center Consensus Conference 2017. Eur J Dent 2018;12(4):617-26.

[7] Turkoglu P, Kose A, Sen D. Abutment selection for anterior implant-supported restorations. In: Almasri MA, ed. An update of dental implantology and biomaterial. Intech 
Open 2019. https://www.intechopen.com/books/anupdate-of-dental-implantology-and-

biomaterial/abutment-selection-for-anterior-implantsupported-restorations

[8] Gupta A, Jain D, Sangwan A, et al. Abutment Options for Compromised situation in aesthetic zone- case reports. IOSR J Dent Med Sci (IOSR-JDMS) 2015;14(8):30-7.

[9] Patel V, Khandelwal M, Punia V, et al. Single implant supported fixed prosthesis in anterior zone: a review. Int J Appl Dent Sci 2018;4(2):299-304.
[10] Ahmad M, Dhanasekar B, Aparna IN, et al. Replacement of missing anterior tooth using screw retained implant prosthesis in the aesthetic zone: a case report with 3 years of follow up. J Indian Prosthodont Soc 2014;14(3):297300.

[11] Shadid R, Sadaqa N. A Comparison between screw- and cement-retained implant prostheses. A literature review. J Oral Implantol 2012;38(3):298-307. 